eISSN 2444-7986

DOI: https://doi.org/10.14201/orl.21241

ARTÍCULO DE REVISIÓN

\title{
REHABILITACIÓN VESTIBULAR EN LA DEPENDENCIA VISUAL Y SOMATOSENSORIAL
}

\section{Vestibular rehabilitation for visual and somatosensory dependency}

\author{
Michael BAUER; José Ignacio BENITO-OREJAS; Jesús Eduardo RAMÍREZ-SALAS \\ SACYL. Hospital Clínico Universitario de Valladolid. Servicio de Otorrinolaringología y CCF. Valladolid. España. \\ Correspondencia: jbenitoorejas@gmail.com
}

Fecha de recepción: 5 de agosto de 2019

Fecha de aceptación: 26 de septiembre de 2019

Fecha de publicación: 28 de septiembre de 2019

Fecha de publicación del fascículo: 1 de marzo de 2020

Conflicto de intereses: Los autores declaran no tener conflictos de intereses

Imágenes: Los autores declaran haber obtenido las imágenes con el permiso de los pacientes

Política de derechos y autoarchivo: se permite el autoarchivo de la versión post-print (SHERPA/RoMEO)

Licencia CC BY-NC-ND. Licencia Creative Commons Atribución-NoComercial-SinDerivar 4.0 Internacional

Universidad de Salamanca. Su comercialización está sujeta al permiso del editor

RESUMEN: Introducción: En la disfunción vestibular crónica es frecuente que se incremente el mareo o el desequilibrio con el propio movimiento o con el de un ambiente visual sobrecargado, lo que se denomina dependencia visual, que forma parte del síndrome de vértigo/mareo inducido visualmente. En otras ocasiones, la dependencia es somatosensorial y el paciente pierde con facilidad el equilibrio al alterarse la superficie de soporte. El objetivo de esta revisión es describir las características de la dependencia visual y somatosensorial y el modo de tratarlas. Método: Revisión narrativa. Resultados: El vértigo visual se diagnostica clínicamente a través de los síntomas referidos por el paciente. Mediante la exposición a estímulos desencadenantes, el sistema nervioso central se habitúa, incrementando la tolerancia. El tratamiento utilizado se basa en la terapia física, la realidad virtual y la estimulación optocinética progresiva. Conclusión/ Discusión: Aunque sigue siendo tema de discusión la naturaleza de las entradas visuales que determinan la estabilidad postural, el objetivo terapéutico consiste en desensibilizar al individuo del estímulo visual para mejorar su sintomatología.

PALABRAS CLAVE: vértigo visual; rehabilitación vestibular; habituación; equilibrio; dependencia visual; estabilidad postural. 
SUMMARY: Introduction: In the patient with chronic vestibular dysfunction, it is very frequent to note more imbalance or dizziness produced by its own movement or the overloaded visual environment; this is called also visual dependence that is part of the visual vertigo syndrome. In other cases, like somatosensory dependence, the patient will lose balance easily when the support surface changes. The objective of this review was to describe the visual and somatosensory dependence patterns as well as how to treat them. Method: Narrative review. Results: Visual vertigo is currently a clinical diagnosis based on the patient's history of disease presentation. Through repeated exposure to trigger stimuli, the central nervous system is able to increase tolerance and improve functional results. Traditional treatments for this disorder involve vestibular rehabilitation therapy, virtual reality simulators of moving objects, as well as graded exposure to optokinetic stimulation. Discussion/Conclusion: Although the real nature of the visual cues for posture stabilization remains an open debate, the goal of therapy is to promote desensitization to visual stimuli and increase tolerance.

KEYWORDS: visual vertigo; vestibular rehabilitation; habituation; balance; visual dependence; postural stability.

\section{INTRODUCCIÓN}

\section{DEPENDENCIA VISUAL. CONCEPTO Y APROXIMACIÓN FISIOPATOLÓGICA}

La interacción entre los sistemas visual y vestibular es consecuencia de la convergencia anatómica y funcional de las señales que proceden de ambos. La visión puede incrementar o suprimir (supresión del reflejo vestíbulo-ocular) la respuesta vestibular, pero, además, desarrolla una importante función en el control postural, con una influencia sinérgica sobre los reflejos posturales vestíbulopropioceptivos, lo que se evidencia, por ejemplo, al observar el incremento del balanceo del cuerpo cuando se cierran los ojos [1]. Sin embargo, en determinadas circunstancias, el sistema visual y el vestibular provocan efectos opuestos sobre la postura. Así, cuando el campo visual se mueve o se proyectan imágenes en movimiento, se produce una oscilación o inclinación corporal hacia donde se dirige el estímulo visual [2]. En el primer siglo de nuestra era, Sorano de Éfeso ya describe el efecto de inestabilidad que se experimenta al fijar la vista en la corriente del agua o en la rueda del torno del alfarero, mientras gira [3]. La intensidad de estas sensaciones varía mucho en la población general.
Todos hemos experimentado la ilusión de creer que nuestro tren es el que se desplaza (vección), cuando lo hace el de al lado o de tener que apretar el freno porque parados en una rampa, el coche de delante se viene hacia nosotros, al suponer que somos nosotros quienes nos abalanzamos sobre él. Esta ambigüedad visual, desde el punto de vista de la orientación espacial, se suma a la información que aportan al sistema nervioso central (SNC) los sistemas sensoriales basados en la inercia (sistema vestibular y propioceptivo), que no confirman lo que el sistema visual malinterpreta como un movimiento del cuerpo. A través de determinados mecanismos neurales se analiza el grado de acuerdo que existe entre las diferentes señales y si no son proporcionales, se genera una reacción de alerta que desencadena un acción automática o voluntaria (por ej., mirar a un punto estacionario para comprobar si somos nosotros o es el tren el que se mueve). En definitiva, en función de las circunstancias, el SNC determina la importancia (el peso relativo) de cada una de estas tres informaciones sensoriales y de la misma manera, en caso de enfermedad, el SNC es quien se responsabiliza de compensar el déficit sensorial (compensación central) [4]. 
Hay pacientes con déficit vestibular o propioceptivo y algunos con alteraciones del SNC que se sienten más inestables que otros, durante el movimiento de escenas visuales [5-7]. Tras un déficit vestibular, la recuperación de la estabilidad postural es más lenta que la de la mirada [8]. Aunque a través de la adaptación, intentaremos mejorar la respuesta vestibular para restablecer el control postural, resultan fundamentales los mecanismos de preferencia visual y somatosensorial (sustitución) [9]. En caso de déficits temporales, se reponen las estrategias posturales normales, pero si la alteración vestibular es crónica son necesarias otras maniobras compensatorias.

La orientación espacial y el control postural dependen de las referencias vestíbulo-espinal y visual y las personas sanas utilizan de forma variable e idiosincrásica cada una de estas entradas; en presencia de un conflicto sensorial, hay sujetos que confían más en la entrada visual y otros que se apoyan principalmente en la vestíbulo-espinal. El término utilizado para describir un aumento de respuesta a la orientación o al movimiento de un estímulo visual, es el de dependencia visual. Es probable que estas preferencias perceptuales observadas en sujetos normales estén también presentes e incluso incrementadas, en pacientes con alteraciones del equilibrio [5]. Pacientes dependientes visuales serían aquellos que se apoyan de forma anormal en la información visual para mantener el equilibrio, persistiendo en su uso, aunque dicha información sea incorrecta y/o entre en conflicto con el resto de la información sensorial. Si la vertical visual no está alineada con la gravedad, el paciente (sobre todo en superficies inestables) desplazará el cuerpo de acuerdo con la inclinación del eje visual y se desestabilizará [10]. De forma similar una escena visual en movimiento puede ser malinterpretada como un movimiento del propio cuerpo e inducir un ajuste postural corrector que cause inestabilidad [11].

El conflicto sensorial es similar al que Brandt et al, 1980 [12] describen en el vértigo fisiológico de las alturas, como consecuencia de la disparidad visuovestibular que se produce por la excesiva distancia entre el observador y el contraste visible estacionario. En condiciones normales, a medida que aumenta la distancia entre el sujeto y la diana, se necesita incrementar la amplitud del balanceo para percibir visualmente el movimiento. El vértigo de altura se desencadena como resultado de esta incongruencia. El sistema vestibular y el somatosensorial distinguen el balanceo corporal, pero el visual no capta el movimiento del objeto lejano, lo que acrecienta a su vez el balanceo, la posibilidad de desequilibrio y la sensación vertiginosa que puede incrementarse por factores psicológicos, responsables de la ansiedad y de la aparición de síntomas vegetativos [7].

Cuando un individuo es visualmente dependiente, una escena en movimiento (paso de coches en una calle o escenas de persecución en una película, desplazamiento de gente, de cortinajes, de hojarasca con el viento, de nubes al mirar hacia el cielo, de luces en la discoteca o de los árboles de la carretera conduciendo en un día soleado) o muy sobrecargada visualmente (al caminar por los pasillos de un supermercado mirando los múltiples objetos situados en los anaqueles (síndrome del supermercado [13])), e incluso, en algunos pacientes, el simple movimiento de los ojos, la lectura o el parpadeo de un fluorescente, pueden causarles una sensación de seudoebriedad o ser malinterpretado como movimiento del propio cuerpo, induciendo ajustes posturales que produzcan desequilibrio [7] y requieran de tratamiento $[9,14-16]$.

Aunque el término de vértigo visual fue originariamente utilizado por Erasmus Darwin en 1797 [13], Bronstein lo reintroduce en 1995 [17], describiéndolo como un síndrome que puede aparecer en aquellos pacientes con alteración vestibular que desarrollan dependencia visual. En lengua anglosajona también se conoce como visual vestibular mismatch (desajuste visuovestibular) [13] y el Comité Internacional de Alteraciones Vestibulares de la Sociedad Bárány lo denomina, vértigo/ 
mareo inducido visualmente y lo define como el síntoma que se desencadena por un estímulo visual complejo, distorsionado, de amplio campo o en movimiento, incluyendo el movimiento relativo del entorno visual respecto al propio cuerpo. También comprende aquellos casos en los que el input visual que causa el vértigo se origina en una alteración de la motilidad ocular primaria (por ejemplo, en una mioquimia de un músculo ocular o en un nistagmo no vestibular) [18]. Este síntoma no se debe confundir con la oscilopsia, en el que se produce una falsa sensación de oscilación del entorno visual. Es pues un síntoma visual, mientras que, en el vértigo/mareo inducido visualmente, el desencadenante es visual, pero el síntoma es vestibular: mareo, vértigo o desequilibrio [19].

\section{MANIFESTACIONES CLÍNICAS}

El vértigo visual que aparece tras una alteración vestibular (por ej., una neuritis vestibular o más infrecuentemente tras un vértigo posicional paroxístico benigno o la inyección intratimpánica de gentamicina [13]) suele manifestarse clínicamente porque el paciente, después de un periodo de recuperación de unas semanas, nota que el mareo no sólo no desaparece, sino que empieza a agravarse en entornos visuales conflictivos (caminar por el pasillo de un supermercado, contemplar la pantalla de un cine, etc.). Con frecuencia se produce, además, ansiedad o frustración, porque persisten los síntomas o porque el médico les resta importancia [4].

Esta hiperreactividad visuopostural también se puede experimentar, en casos de alteración vestibular central, en la migraña, en la ansiedad y en pacientes que combinan una alteración vestibular con un estrabismo congénito o una cirugía del estrabismo [17].

Ediciones Universidad de Salamanca / @@@

\section{VALORACIÓN DIAGNÓSTICA}

Se desconoce por qué un paciente concreto, tras una alteración vestibular, desarrolla esta dependencia visual [17] y no está claro si se trata de un fenómeno que afectaría únicamente a pacientes susceptibles [20]. Tampoco se sabe si la ansiedad/ depresión que suele acompañarla, es un fenómeno primario o secundario, aunque hasta el momento no hay evidencias de que los niveles de ansiedad/ depresión sean diferentes a los que manifiestan otros pacientes con vértigo [5, 21]. En cualquier caso, este componente psicológico que invariablemente se añade a cualquier patología vestibular y donde resulta muy difícil reconocer si es previo o posterior, quizá debiera recibir un tratamiento específico, antes incluso de iniciar la rehabilitación [22]. De acuerdo con Bronstein AM, 2013 [4, 19], la dependencia visual, las alteraciones vestibulares asociadas y el vértigo visual son tres conceptos tan relacionados, que resulta difícil saber cuál fue antes «si el huevo o la gallina» [4].

El diagnóstico diferencial más complejo se genera con los ataques de pánico [23]. No se dispone de un conjunto de criterios que permitan establecer la diferencia; pero un paciente sin una clara historia de enfermedad vestibular, sin hallazgos exploratorios de alteración vestibular $\mathrm{y}$ con desencadenantes visuales restringidos a un determinado ambiente (por ej., sólo le ocurre en supermercados), es más probable que tenga una alteración psicológica. Por el contrario, un individuo sin antecedentes de trastorno psíquico, que después de una disfunción vestibular desarrolla una dependencia visual, es posible que tenga un síndrome de vértigo/mareo inducido visualmente [19].

Los niños son más susceptibles que los adultos al mareo inducido visualmente, al ser más dependientes de las entradas visuales y disponer de un sistema encargado de resolver el conflicto sensorial más inmaduro, que no alcanza su pleno desarrollo hasta la adolescencia [24]. Es importante que los 


\section{REHABILITACIÓN VESTIBULAR EN LA DEPENDENCIA VISUAL Y SOMATOSENSORIAL}

BAUER M ET AL

profesionales reconozcan estos síntomas en un niño con migraña o tras una contusión cerebral y/o alteración vestibular, para poder aplicar un tratamiento [24].

Desde el punto de vista práctico el problema consiste en identificar la presencia de dependencia visual en un paciente con mareo crónico. Para ello podemos valernos de la información clínica, de la exploración [25] y de determinados cuestionarios, como la Escala Analógica para el Vértigo Visual (conocida en la literatura anglosajona como VVAS - Visual Vertigo Analogue Scale - [26-27]), Tabla 1.

El diagnóstico permite, como veremos a continuación, la aplicación de un tratamiento específico y adaptado a la situación del paciente.

\section{TRATAMIENTO DE LA DEPENDENCIA VISUAL}

Mediante la rehabilitación vestibular (RV) el paciente aprende a potenciar las referencias visuales y la información somatosensorial, como sistemas primarios de información sensorial. Pero resulta fundamental de cara al pronóstico, conocer si la afectación vestibular es uni o bilateral, completa o no y si hay otras alteraciones sensoriales [11].

Se ha demostrado que la terapia física reduce el vértigo/mareo inducido visualmente [28-31]. Los ejercicios preferidos en estos casos son los de habituación [32], que consisten en aplicar de forma gradual y repetitiva, movimientos o situaciones que provoquen síntomas, con la finalidad de desensibilizar al paciente e incrementar su tolerancia al estímulo visual y al conflicto visuovestibular.

Se propondrán ejercicios de equilibrio con una entrada visual reducida (ojos cerrados) o distorsionada (semipenumbra, gafas de sol) y con un buen input somatosensorial (por ej., con los pies descalzos sobre una superficie estable) [11] y se prevendrá al paciente de que los realice con la vista al frente, pues se tiende a mirar al suelo para evitar el mareo [19].

Ediciones Universidad de Salamanca / @@@
Tabla 1. Escala visual analógica de vértigo. Escala traducida y modificada para valorar el vértigo visual.

(Dannenbaum et al. 2011) [28]

Indique el mareo/vértigo o desequilibrio que Vd. podría experimentar en las siguientes situaciones, puntuándolas de «0» (nada de mareo/vértigo o desequilibrio) a «10» puntos (máximo mareo/vértigo o desequilibrio):

1. Yendo de pasajero en un coche:

2. Estando bajo tubos fluorescentes:

3. Mirando el tráfico en un cruce:

4. Caminando por un supermercado:

5. Estando en un centro comercial:

6. En una escalera mecánica:

7. Viendo una película en el cine:

8. Caminando sobre un suelo con dibujos:

9. Mirando la televisión:

El paciente mostrará positividad al vértigo visual si 20 más items se puntúan por encima de 0 .

El grado de severidad se expresa en porcentaje respecto al máximo posible.

Pero también se pueden utilizar la realidad virtual u otros procedimientos de inmersión [28, 33-34] y el estímulo optocinético (OKN) [29-30] (ver capítulo referido a nuevas tecnologías en rehabilitación vestibular, Álvarez-Otero, R). La realidad virtual introduce al paciente en un ambiente visual conflictivo, mientras que el estímulo $\mathrm{OKN}$ consiste en el uso repetitivo de patrones en movimiento que pueden generase de forma muy diversa: a través de video proyecciones (DVD) [35], sistemas planetarios o de rotación OKN [36].

El desarrollo de la industria del juego ha conseguido una realidad virtual de bajo precio, con equipos como la Nintendo Wii Fit Plus que incluye una plataforma que permite establecer un feedback con la pantalla, durante los juegos de equilibrio [37]. No hay diferencias en los resultados obtenidos con la Wii Fit y los ejercicios convencionales de equilibrio [29], pero en general, a los pacientes les resulta más divertido el entrenamiento virtual [38]. Otros métodos más económicos de estimulación consisten en el uso de discos rotatorios con círculos de diferentes tamaños y colores, giro de paraguas, movimiento de grandes cartulinas con líneas o un 
simple mantel de cuadros colocado en la pared, donde hay que ir buscando diferentes números [4, 8]. Los vídeos de acción se pueden conseguir de forma directa en YouTube [8] y el uso de estímulos OKN obtenidos a través de un DVD [35], resulta un procedimiento económico y de fácil aplicación en un programa de RV.

Se ha demostrado que la supervisión facilita el cumplimiento, la mejora de la estabilidad postural y del estado psicológico, frente a una práctica no supervisada [29].

El tratamiento de habituación para la dependencia visual requiere breves dosis de estímulo visual, que provoquen síntomas de leves a moderados (por ej., ver un vídeo de acción durante 1 minuto), seguido por un periodo de descanso, antes de introducir la siguiente estimulación [8]. También la progresión se puede realizar pasando de una pantalla pequeña a otra más grande. El paciente comienza mirando estos estímulos sentado, luego de pie, caminando hacia adelante o hacia atrás; inicialmente sin y posteriormente con movimientos de cabeza, de una forma progresiva $[8,11]$, mejorando a través de este programa de entrenamiento tanto el mareo como los síntomas psicológicos asociados [19].

Los pacientes que sufren de migraña pueden ser sensibles a su propio movimiento y al del ambiente y en estos casos la terapia debe ser muy lenta para no desencadenar dolor de cabeza o nausea y los ejercicios de habituación nunca se deben realizar durante la cefalea [8]. Hay quienes consideran que, en la migraña vestibular, las crisis de jaqueca podrían disminuir o controlarse mejor con rehabilitación visuovestibular [39-40].

Informaremos al paciente que durante la primera semana va a experimentar más síntomas, pues está realizando movimientos que en condiciones normales intenta evitar. Smith-Wheelock et al. [41] sugieren que estos ejercicios de habituación se hagan al principio sólo 2 veces al día, para evitar la sobrecarga del sistema compensatorio. Realizaremos la reevaluación al mes de iniciada la terapia, pues se ha comprobado que la mejoría se produce entre las 4 y las 6 semanas [8].

Finalmente, hay que mencionar que se ha especulado con el uso de la acetazolamida (inhibidor de la anhidrasa carbónica) en el vértigo visual, con una dosis media de $250 \mathrm{mg}$ /día, que con escasos efectos secundarios podría aliviar la sintomatología. Se desconoce si su efecto diana es sobre el vestíbulo, disminuyendo la presión hidrostática o en el centro cerebeloso responsable de procesar la información visual [42-43].

\section{DEPENDENCIA SOMATOSENSORIAL}

\section{Concepto}

Suele aparecer durante la recuperación vestibular, especialmente en caso de déficits bilaterales, pero también puede ocurrir en patología otolítica o en alteraciones del equilibrio de origen central [44]. En contraste con quienes sufren una hipofunción unilateral, el paciente con déficit bilateral confía sobre todo en la entrada visual en el estadio agudo y en la somatosensorial en la fase crónica [45-46]. Puede ocurrir que, en esta situación, la entrada propioceptiva se erija en la más importante para ofrecer las señales de error requeridas por los núcleos vestibulares para conseguir mantener el equilibrio estático [47]. Este fenómeno se conoce como dependencia somatosensorial [11].

\section{Tratamiento de LA DEPENDENCiA} SOMATOSENSORIAL

Como en el supuesto de la dependencia visual, se trata de que el paciente aprenda a utilizar de forma coordinada las informaciones sensoriales, interpretando y calibrando de manera apropiada sus señales y estableciendo respuestas adecuadas a las mismas.

Para superarlo, el paciente realizará ejercicios, sentado o de pie, sobre superficies inestables, tales como alfombras, gomaespuma, plataformas móviles (por ej. un balancín) o planos inclinados (rampas). 
Se le puede entrenar también, con juegos de pelota mientras se encuentra con los pies sobre una alfombra u otra superficie irregular [11].

Adaptaremos el medio a la seguridad, sin que tenga que ser necesaria la continua supervisión del terapeuta [11]. Y así, a los pacientes inestables o con miedo a caerse, les colocaremos un arnés, indicaremos que los ejercicios de equilibrio estático se realicen cerca de una pared o frente a una mesa o silla para poderse sujetar y los ejercicios dinámicos pueden desarrollarse entre barras paralelas para sentirse más seguros [11].

Los pacientes con dependencia sensorial que no responden a los ejercicios de habituación suelen ser, los que describen síntomas vagos de mareo que no se modifican con el movimiento ambiental, los que no siguen las pautas prescritas y aquellos donde la causa es de origen psíquico. Cuando no mejora la sintomatología o incluso empeora a pesar de adaptar la terapia, se debe replantear el diagnóstico [8].

\section{CONCLUSIONES}

Los estímulos sensoriales que hacen posible la orientación espacial y el equilibrio provienen del sistema visual, vestibular y somatosensorial. En condiciones normales los tres se solapan y actúan de forma sinérgica y congruente, coordinados por el SNC que reparte la relevancia de cada uno en función de las circunstancias del medio, de la experiencia adquirida y del estado emocional. De la misma manera, en presencia de una lesión vestibular, los mecanismos de compensación central reequilibran el peso de cada input. Pero en ocasiones, circunstancias fisiológicas (vértigo de las alturas, cinetosis [48], dependencia visual, etc.) o patológicas, favorecen el que estos sistemas entren en conflicto. La presencia de ambientes sensoriales complejos (no familiares o ambiguos), pueden afectar al sistema vestibular en función de la susceptibilidad individual y de factores de tipo psicológico. Si el sistema vestibular está alterado, estos síntomas (vértigo, desequilibrio y cuadro vegetativo) pueden aparecer incluso en situaciones cotidianas.

En este capítulo nos hemos centrado específicamente en la dependencia visual que se desarrolla en algunos pacientes con afectación vestibular (mareo inducido visualmente o vértigo visual), quienes refieren un empeoramiento de su sintomatología frente a un estímulo visual en movimiento. Se desconoce la causa por la que se desarrolla esta dependencia, que también se puede experimentar en casos de alteración vestibular central, en la migraña, etc. [17]. El tratamiento fundamental se basa en la desensibilización progresiva [19].

\section{AGRADECIMIENTOS}

A quien ha tenido a bien corregir nuestro trabajo, por su esfuerzo y comentarios. Nos ha permitido aprender más y mejorar la calidad de este manuscrito. Creemos que la arriesgada labor de revisión, que conlleva responsabilidad, trabajo y pocas satisfacciones, debería ser reconocida y recompensada con la excelencia.

\section{BIBLIOGRAFÍA}

1. Bronstein AM. Vision and vertigo: some visual aspects of vestibular disorders. J Neurol. 2004 Apr;251(4):381-7.

2. Dichgans J, Held R, Young LR, Brandt T. Moving visual scenes influence the apparent direction of gravity. Science. 1972 Dec 15;178(4066):1217-9.

3. Balaban CD, Jacob RG. Background and history of the interface between anxiety and vertigo. J Anxiety Disord. 2001 Jan-Apr;15(1-2):27-51.

4. Bronstein AM. Multisensory integration in balance control. En: Aminoff MJ, Boller F, Swaab D. Handbook of clinical neurology. Neuro-Otology. Furman JM, Lempert T eds. Vol. 137 (3rd series). Elsevier;2016. p.57-66.

5. Guerraz M, Yardley L, Bertholon P, Pollak L, Rudge P, Gresty MA, et al. Visual vertigo: symptom 


\section{REHABILITACIÓN VESTIBULAR EN LA DEPENDENCIA VISUAL Y SOMATOSENSORIAL BAUER M ET AL}

assessment, spatial orientation and postural control. Brain. 2001 Aug;124(Pt 8):1646-56.

6. Peterka RJ, Benolken MS. Role of somatosensory and vestibular cues in attenuating visually induced human postural sway. Exp Brain Res. 1995;105(1):101-10.

7. Pérez-Fernández N, Fernández-González S. Vértigo ocular. En: Bartual-Pastor J, Pérez-Fernández N. El sistema vestibular y sus alteraciones. Tomo II. $1^{\text {a }}$ ed. Barcelona: Masson, SA;1999. p.495-500.

8. Herdman SJ, Whitney SL. Physical therapy treatment of vestibular hypofunction. En: Herdman SJ, Clendaniel RA. Vestibular Rehabilitation. $4^{\mathrm{a}} \mathrm{ed}$. Philadelphia: FA Davis Co;2014. p.394-431.

9. Herdman SJ. Advances in the treatment of vestibular disorders. Phys Ther. 1997 Jun;77(6):602-18.

10. Horak FB. Postural compensation for vestibular loss and implications for rehabilitation. Restor Neurol Neurosci. 2010;28(1):57-68. doi: 10.3233/ RNN-2010-0515.

11. Han BI, Song HS, Kim JS. Vestibular rehabilitation therapy: review of indications, mechanisms, and key exercises. J Clin Neurol. 2011 Dec;7(4):184-96. doi: 10.3988/jcn.2011.7.4.184.

12. Brandt T, Arnold F, Bles W, Kapteyn TS. The mechanism of physiological height vertigo. I. Theoretical approach and psychophysics. Acta Otolaryngol. 1980 May-Jun;89(5-6):513-23.

13. Longridge NS, Mallinson AI. Visual vestibular mismatch in work-related vestibular injury. Otol Neurotol. 2005 Jul;26(4):691-4.

14. Hoffman RA, Brookler KH. Underrated neurotologic symptoms. Laryngoscope. 1978 Jul;88(7 Pt 1):1127-38.

15. Hood JD. Unsteadiness of cerebellar origin: an investigation into its cause. J Laryngol Otol. 1980 Aug;94(8):865-76.

16. Bronstein AM. Under-rated neuro-otological symptoms: Hoffman and Brookler 1978 revisited. Br Med Bull. 2002;63:213-21.

17. Bronstein AM. Visual vertigo syndrome: clinical and posturography findings. J Neurol Neurosurg Psychiatry. 1995 Nov;59(5):472-6.
18.Bisdorff A, Von Brevern M, Lempert T, NewmanToker DE. Classification of vestibular symptoms: towards an international classification of vestibular disorders. J Vestib Res. 2009;19(1-2):1-13. doi: 10.3233/VES-2009-0343.

19. Bronstein AM. Oscillopsia and visuo-vestibular symptoms. En: Bronstein AM. Oxford textbook of Vertigo and Imbalance. $1^{\text {a }}$ ed. United Kingdom: Oxford University Press;2013. p.137-44.

20. Van Ombergen A, Lubeck AJ, Van Rompaey V, Maes LK, Stins JF, Van de Heyning PH, et al. The Effect of Optokinetic Stimulation on Perceptual and Postural Symptoms in Visual Vestibular Mismatch Patients. PLoS One. 2016 Apr 29;11(4):e0154528. doi: 10.1371/journal. pone. 0154528.

21. Pavlou M, Davies RA, Bronstein AM. The assessment of increased sensitivity to visual stimuli in patients with chronic dizziness. J Vestib Res. 2006;16(4-5):223-31.

22. Bronstein AM, Lempert T. Management of the patient with chronic dizziness. Restor Neurol Neurosci. 2010;28(1):83-90. doi: 10.3233/ RNN-2010-0530.

23. Furman JM, Jacob RG. Psychiatric dizziness. Neurology. 1997 May;48(5):1161-6.

24. Pavlou M, Whitney SL, Alkathiry AA, Huett M, Luxon LM, Raglan E, et al. Visually Induced Dizziness in Children and Validation of the Pediatric Visually Induced Dizziness Questionnaire. Front Neurol. 2017 Dec 5;8:656. doi: 10.3389/fneur.2017.00656.

25. Cousins S, Cutfield NJ, Kaski D, Palla A, Seemungal BM, Golding JF, et al. Visual dependency and dizziness after vestibular neuritis. PLoS One. 2014 Sep 18;9(9):e105426. doi: 10.1371/journal. pone.0105426. eCollection 2014.

26. Dannenbaum E, Chilingaryan G, Fung J. Visual vertigo analogue scale: an assessment questionnaire for visual vertigo.J Vestib Res. 2011;21(3):153-9. doi: 10.3233/VES-2011-0412.

27. Dannenbaum E, Chilingarian G, Fung J. Validity and Responsiveness of the Visual Vertigo Analo- 


\section{REHABILITACIÓN VESTIBULAR EN LA DEPENDENCIA VISUAL Y SOMATOSENSORIAL BAUER M ET AL}

gue Scale. J Neurol Phys Ther. 2019 Apr;43(2):117121. doi: 10.1097/NPT.0000000000000261.

28. Pavlou M, Kanegaonkar RG, Swapp D, Bamiou DE, Slater M, Luxon LM. The effect of virtual reality on visual vertigo symptoms in patients with peripheral vestibular dysfunction: a pilot study. J Vestib Res. 2012;22(5-6):273-81. doi: 10.3233/VES-120462.

29. Pavlou M, Bronstein AM, Davies RA. Randomized trial of supervised versus unsupervised optokinetic exercise in persons with peripheral vestibular disorders. Neurorehabil Neural Repair. 2013 Mar-Apr;27(3):208-18. doi: $10.1177 / 1545968312461715$.

30. Pavlou M. The use of optokinetic stimulation in vestibular rehabilitation. J Neurol Phys Ther. 2010 Jun;34(2):105-10. doi: 10.1097/ NPT.0b013e3181dde6bf.

31. Szturm T, Ireland DJ, Lessing-Turner M. Comparison of different exercise programs in the rehabilitation of patients with chronic peripheral vestibular dysfunction. J Vestib Res. 1994 Nov-Dec;4(6):461-79.

32. Sulway S, Whitney SL. Advances in Vestibular Rehabilitation. Adv Otorhinolaryngol. 2019;82:164-169. doi: 10.1159/000490285.

33. Gottshall KR, Sessoms PH, Bartlett JL. Vestibular physical therapy intervention: utilizing a computer assisted rehabilitation environment in lieu of traditional physical therapy. Conf Proc IEEE Eng Med Biol Soc. 2012;2012:6141-4. doi: 10.1109/ EMBC.2012.6347395.

34. Alahmari KA, Sparto PJ, Marchetti GF, Redfern MS, Furman JM, Whitney SL. Comparison of virtual reality based therapy with customized vestibular physical therapy for the treatment of vestibular disorders. IEEE Trans Neural Syst Rehabil Eng. 2014 Mar;22(2):389-99. doi: 10.1109/TNSRE.2013.2294904.

35. Trinidad Ruiz G, Domínguez Pedroso M, Cruz de la Piedad E, Solís Vázquez R, Samaniego Regalado B, Rejas Ugena E. Rehabilitación vestibular domiciliaria guiada, asistida por medios audiovisuales. Acta Otorrinolaringol
Esp. 2010 Nov-Dec;61(6):397-404. doi: 10.1016/j. otorri.2010.07.003.

36. Vitte E, Sémont A, Berthoz A. Repeated optokinetic stimulation in conditions of active standing facilitates recovery from vestibular deficits. Exp Brain Res. 1994;102(1):141-8.

37. Clark RA, Bryant AL, Pua Y, McCrory P, Bennell K, Hunt M. Validity and reliability of the Nintendo Wii Balance Board for assessment of standing balance. Gait Posture. 2010 Mar;31(3):307-10. doi: 10.1016/j.gaitpost.2009.11.012.

38. Meldrum D, Herdman S, Vance R, Murray D, Malone K, Duffy D, et al. Effectiveness of conventional versus virtual reality-based balance exercises in vestibular rehabilitation for unilateral peripheral vestibular loss: results of a randomized controlled trial. Arch Phys Med Rehabil. 2015 Jul;96(7):1319-1328.e1. doi: 10.1016/j. apmr.2015.02.032.

39. Sugaya N, Arai M, Goto F. The effect of vestibular rehabilitation on sleep disturbance in patients with chronic dizziness. Acta Otolaryngol. 2017 Mar;137(3):275-278. doi: 10.1080/00016489.2016.1244859.

40. Alghadir AH, Anwer S. Effects of Vestibular Rehabilitation in the Management of a Vestibular Migraine: A Review. Front Neurol. 2018 Jun 12;9:440. doi: 10.3389/fneur.2018.00440.

41. Smith-Wheelock M, Shepard NT, Telian SA. Physical therapy program for vestibular rehabilitation. Am J Otol. 1991 May;12(3):218-25.

42. Hester RB 3rd, Farris BK. Acetazolamide in the treatment of abnormal oculovestibular response. Am J Ophthalmol. 1991 Feb 15;111(2):215-20.

43. Sluch IM, Elliott MS, Dvorak J, Ding K, Farris BK. Acetazolamide: A New Treatment for Visual Vertigo. Neuroophthalmology. 2017 Aug 2;41(6):315-320. doi: 10.1080/01658107.2017.1326944.

44. Sauvage JP. Rehabilitation vestibulaire: bilan et méthodes. En: Sauvage JP. Vertiges: manuel de diagnostic et de réhabilitation. $1^{\text {a }}$ ed. Paris: Elsevier Masson;2010. p.137-46. 
45. Herdman SJ. Role of vestibular adaptation in vestibular rehabilitation. Otolaryngol Head Neck Surg. 1998 Jul;119(1):49-54.

46. Bles W, Vianney de Jong JM, de Wit G. Compensation for labyrinthine defects examined by use of a tilting room. Acta Otolaryngol. 1983 May-Jun;95(5-6):576-9.
47. Zee DS. Vestibular adaptation. En: Herdman SJ. Vestibular Rehabilitation. $3^{\text {a }}$ ed. Philadelphia: FA Davis Co;2007. p.77-90.

48. Sanchez-Blanco C, Yañez-Gonzalez R, BenitoOrejas JI, Gordon CR, Batuecas-Caletrio A. Cinetosis. Rev Soc Otorrinolaringol Castilla Leon Cantab La Rioja. 2014 Nov. 5 (28): 233-251. 